\title{
Interaction génotype-densité et compétition dans un dispositif clinal d'épicéa commun
}

\author{
P Dreyfus
}

INRA, Station de sylviculture méditerranéenne, avenue Antonio-Vivaldi, 84000 Avignon, France

(Reçu le 6 mai 1988; accepté le 16 février 1989)

\begin{abstract}
Résumé - Dans le nord-est de la France, 2 descendances maternelles d'épicéa commun (Picea abies Karst.) sont cultivées en plantation pure et en mélange dans un dispositif expérimental original où densité du peuplement et taux de mélange varient de façon clinale (densité 200-10 000/ha, taux de mélange $0 \%-100 \%$ ). La descendance GANN 7 est nettement plus vigoureuse que MORZ 4. Pour les 2 génotypes, la circonférence atteinte 14 ans après la plantation (hauteur moyenne 5 à $8 \mathrm{~m}$ ) est fortement influencée par le gradient de densité, mais l'étude des 2 peuplements purs démontre qu'il n'existe pas pour cette variable d'interaction statistique génotype-densité suffisamment prononcée pour être mise en évidence dans cette expérience. La diminution de taille due à une densité plus forte est la même en valeur absolue pour les 2 génotypes. En mélange, et pour une densité donnée, la croissance d'un arbre, quel que soit son génotype, est d'autant meilleure qu'il se trouve dans une partie du dispositif où la proportion de MORZ 4 est plus forte. MORZ 4 possède donc un pouvoir concurrentiel plus faible que celui de GANN 7, ce qui concorde avec les caractéristiques de vigueur des deux génotypes. Cette tendance est d'autant plus marquée que la densité du peuplement est élevée. On démontre que la forte croissance des individus GANN 7 situés dans une partie à dominance de MORZ 4 est due à la faiblesse de la concurrence exercée par MORZ 4 et non à un phénomène de coopération. L'estimation de la surface terrière de peuplements fictifs composés des deux génotypes étudiés démontre que le peuplement pur du génotype le plus vigoureux (GANN 7) est supérieur aux mélanges. Interaction génotype-densité en peuplement pur et compétition intergénotypique en peuplements mélangés doivent être prises en compte dans la détermination des courbes de croissance, surtout pour des génotypes à faible variabilité génétique.
\end{abstract}

Picea abies / épicéa commun / interaction / génotype / densité / compétition / dispositif clinal / descendance maternelle

Summary - Spacing-genotype interaction and competition in Norway spruce. In the NorthEast of France 2 open-pollinated half-sib progenies (GANN 7 and MORZ 4) of Norway Spruce (Picea abies Karst.) were planted in pure and mixed stands with a special experimental design: clinally varying stand density (200-10 000 stems/ha) and mixture rate (0-100\%). The GANN 7 progeny was much faster growing than MORZ 4. Fourteen years after planting, at age 18 (mean height = 5 to $8 \mathrm{~m}$ ), girth at breast height was strongly reduced by density in both progenies. In pure stands, for girth, the statistical spacing-genotype interaction, if any, was not important enough to be noticed. An absolute value of reduction in girth with increasing density was the same for the two progenies. In mixed stands and for a given density level, the growth of individual trees, whatever their genotype, increased with an increasing proportion of surrounding trees of MORZ 4. Therefore MORZ 4 can be considered as less competitive than GANN 7, which is in accordance with the difference in vigour of the two genotypes. The observed trend was emphasized by increasing stand density. It 
was shown that the fast growth of individual GANN 7 trees surrounded mainly by MORZ 4 trees was only due to the rather weak competition exerted by MORZ 4 and could not be attributed to some kind of cooperation. An estimate of basal area for stands of simulated mixtures of the two genotypes showed clearly that the pure stand of the most vigorous genotype (GANN 7) was superior to all mixed stands. The spacing-genotype interaction in a pure stand and the inter-genotype competition in mixed stands must be taken into account when establishing growth curves, especially for genotypes of very low genetic variability.

Picea abies / Norway spruce / spacing / genotype / interaction / competition / clinal design / half-sib progeny

\section{INTRODUCTION}

Les progrès réalisés en matière de sélection et les perspectives dans ce domaine sont autant de justifications nouvelles pour l'étude du rôle des facteurs génétiques dans la croissance des arbres, dans la compétition et la différenciation sociale au sein des peuplements forestiers. Dans une analyse de l'interface entre amélioration des arbres forestiers et sylviculture, Birot (1986) souligne la nécessité de prendre en compte la dimension génétique dans les recherches sylvicoles et dans la mise au point de modèles de croissance, que ce soit à des fins scientifiques (par exemple pour la recherche sur les phénomènes de compétition) ou pratiques (outils d'aide à la décision destinés aux gestionnaires). Pour certaines variétés sélectionnées, les lois de croissance risquent de s'écarter notablement de celles connues pour des populations ordinaires: relation hauteur dominante-âge de forme ou de niveau différent, accroissement moyen maximum plus élevé ou atteint plus tôt... La réaction à un gradient de densité du peuplement ou à des variations de milieu peut aussi être différente: on parle alors d'interaction génotype-densité ou génotype-milieu. Ceci a d'autant plus de chance de se produire que la variabilité génétique de l'entité considérée est plus faible.
Nous nous intéressons ici à 2 formes d'interaction que nous définissons de manière théorique comme suit:

- l'interaction au sens statistique entre le facteur génotype et la densité du peuplement. Une telle interaction existe lorsque, cultivés séparément, 2 génotypes réagissent différemment à un même gradient de densité. Le long de ce gradient, la différence de croissance entre les 2 génotypes peut s'accroître, diminuer, s'annuler, ou même s'inverser. C'est un cas particulier de l'interaction génotype-environnement. Pour des caractères dendrométriques, Campbell et Wilson (1973) constatent l'absence d'étude rapportant l'existence d'une inversion du classement de 2 génotypes pour des essences forestières; pour des espèces fourragères, le phénomène a été observé entre espèces différentes (graminée et trèfle, par exemple) mais semble absent ou d'importance mineure entre variétés d'une même espèce. Pour 3 provenances de Douglas au stade juvénile, Birot (1972) a démontré l'absence d'une interaction de ce type.

- l'interaction biologique entre génotypes cultivés en mélange, qui existe pratiquement dans tous les peuplements mélangés et qui s'identifie à la «compétition intergénotypique». Elle peut se définir par le fait que la croissance moyenne d'un au moins des composants du mélange est différente de sa croissance moyenne en peuplement pur à la même densité totale. 
Elle résulte des différences entre les caractéristiques intrinsèques d'aptitude à la compétition des génotypes confrontés. Les multiples et souvent infructueuses tentatives visant à cultiver deux ou plusieurs espèces en futaie mélangée ont démontré la difficulté de maintenir la plurispécificité et surtout d'atteindre les objectifs de gestion, du fait de cette interaction interspécifique. Même lorsqu'il s'agit de peuplements naturellement mélangés (chêne-hêtre, hêtre-sapin, sapin-épicéa...), il est difficile de maintenir l'équilibre voulu.

Pour deux clones de peupliers en mélange, Delvaux (1967) a montré qu'il peut exister une corrélation négative entre les 2 caractères de vigueur et de résistance à la concurrence. II suppose même que ceci pourrait être une règle assez générale, tant au niveau spécifique qu'au niveau d'entités génétiques moins variables.

Le maintien d'un mélange au niveau d'équilibre souhaité est d'autant plus difficile que l'existence d'interactions entre le génotype et les conditions de milieu ne permet pas une prévision précise de la croissance des protagonistes, même en peuplement pur.

$\mathrm{Si}$, malgré ces difficultés, des expériences de sylviculture de futaies régulières mélangées ont été poursuivies, c'est souvent dans l'espoir d'obtenir, à densité initiale égale, des productions supérieures à celles des peuplements purs. II s'agirait là d'une des situations décrites dans les travaux de De Wit (1960). En matière forestière et à notre connaissance, cet espoir a toujours été déçu.

Pour Perry (1986), il n'est raisonnable d'escompter un gain substantiel que si les génotypes confrontés sont suffisamment différents. Si tel est le cas, on peut en effet espérer qu'ils exploiteront le milieu dans des compartiments ou à des périodes suffisamment différentes pour qu'ils se concurrencent le moins possible.
Indépendamment de l'aspect production, la culture en peuplement mélangé peut de toute façon présenter des avantages sur le plan phytosanitaire.

II est clair que la densité globale du mélange peut influencer la compétition entre génotypes: à l'extrême, dans un peuplement très lâche, les individus ne sont pas en compétition et n'interagissent pas. Ceci revient à dire qu'il peut exister une interaction statistique entre la densité et l'interaction biologique. C'est probablement pour cette raison que les 2 types d'interaction sont souvent englobés sous le terme unique d'cinteractions génotypedensité».

L'amalgame entre les 2 notions peut cependant rendre délicate l'interprétation de certains résultats expérimentaux. Panetsos (1980) étudie des clones de peupliers cultivés en combinaison au sein d'un dispositif clinal de Nelder, constitué de cercles concentriques. Le facteur densité correspond à l'écartement des plants, de plus en plus grand quand on s'éloigne du centre du dispositif $(\approx 2 \mathrm{~m}$ à $6 \mathrm{~m}$, pour une hauteur à 6 ans de 17 à $20 \mathrm{~m}$ ). Le dispositif est subdivisé en 3 faisceaux de 7 rayons; sur chacun des 7 rayons est installé un clone différent.

Panetsos (1980) obtient un résultat particulièrement intéressant et à peu près unique à notre connaissance: le clone le plus performant à densité faible (vers l'extérieur) n'est pas le même que le plus performant à forte densité (vers le centre), ce qui est interprété en terme d'interaction statistique clone-densité.

Compte tenu de l'espacement des arbres relativement à leur taille, on peut considérer que l'on n'a pas affaire à une comparaison entre peuplements purs. II existe très probablement une interaction biologique (compétition interclones) entre les clones situés sur des rayons adjacents, interaction qui peut varier selon l'espacement. De ce fait, les résultats 
obtenus ne permettent pas de tirer directement des conclusions sur l'opportunité d'utiliser tel ou tel clone selon la densité de plantation pour un peuplement pur; faute de données en peuplement pur, on n'a pas la certitude qu'il existe une interaction statistique clone-densité. Pour les clones considérés, il est possible qu'il y ait à la fois les 2 formes d'interaction, mais peut-être aussi l'une des 2 seulement.

\section{Matériel et Méthode}

L'expérimentation est située en forêt domaniale d'Amance, à $12 \mathrm{~km}$ au nord-est de Nancy (commune d'Amance, Meurthe-et-Moselle). L'altitude avoisine $250 \mathrm{~m}$, le terrain est à peu près horizontal. Le climat, à tendance continentale, comporte encore des influences océaniques marquées. La pluviométrie annuelle moyenne est de $690 \mathrm{~mm}$. Le sol est du type brun lessivé à pseudogley sur marnes liasiques recouvertes de limon; il est assez profond et riche en bases échangeables. L'humus est un mull mésotrophe à eutrophe. Le peuplement d'origine était un taillis-sous-futaie (chêne et charme principalement).

Le dispositif, très original, a été conçu par Décourt (1970). II s'agit d'une parcelle rectan- gulaire, au sein de laquelle sont cultivés en mélange 2 génotypes d'épicéa commun dans des conditions de densité et de mélange qui varient progressivement et de façon continue (caractère clinal) le long des côtés du rectangle. Mis à part les contraintes de densité et de taux de mélange, la disposition des arbres est tirée de façon aléatoire. Le mélange est constitué de 2 descendances maternelles:

- GANN 7, (provenance La Ganne, Auvergne, peuplement artificiel);

- MORZ 4, (provenance Morzine, Préalpes du Nord, autochtone).

Ce mélange était a priori déséquilibré. La provenance La Ganne est très vigoureuse et très précoce; elle peut convenir pour les stations où les gelées tardives ne sont pas trop fréquentes. La provenance Morzine est très précoce et peu vigoureuse à moyenne et basse altitude du fait de sa haute altitude d'origine (1 700-1 $800 \mathrm{~m})$.

Le dispositif comporte une surface expérimentale de $30 \times 60 \mathrm{~m}$, la densité totale $N$ varie de 200 à 10 000/ha (génotype $A+$ génotype $B$ ) dans le sens de la longueur, le pourcentage de plants de type A varie de 0 à $100 \%$ dans le sens de la largeur (fig. 1). On ne note aucune hétérogénéité stationnelle susceptible d'interférer avec les 2 gradients expérimentaux ou de s'y superposer.

Des zones de protection entourent la surface d'expérience sur une largeur de $10 \mathrm{~m}$ (5 m

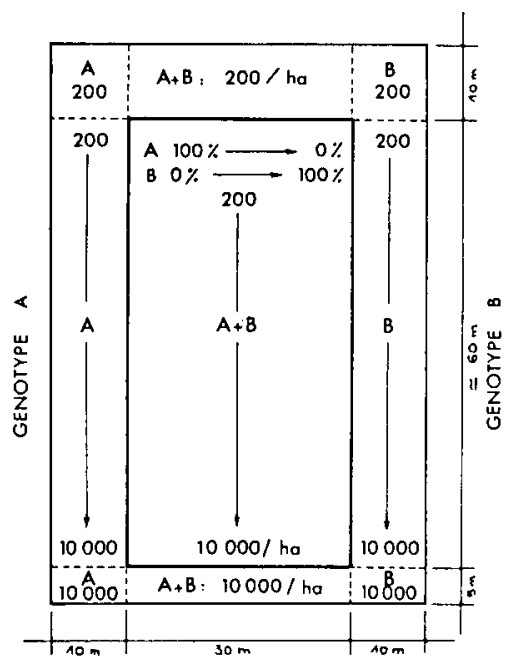

Fig. 1. Structure du dispositif clinal.

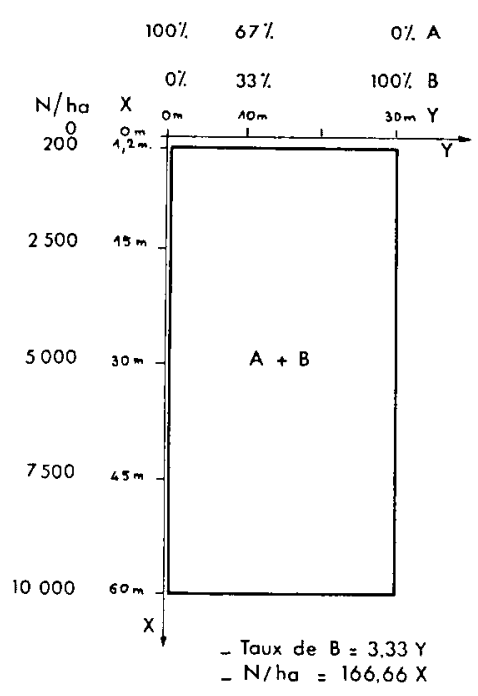


Tableau I. Dimensions moyennes à la plantation et 14 ans après.

\begin{tabular}{lccccc}
\hline & & Moyenne & $\begin{array}{c}\text { GANN 7 } \\
\text { Coeff. de } \\
\text { variation }\end{array}$ & Moyenne & $\begin{array}{c}\text { MORZ 4 } \\
\text { Coeff. de } \\
\text { variation }\end{array}$ \\
\hline $\begin{array}{l}\text { Hauteur à la plantation } \\
\begin{array}{l}\text { Hauteur hors crise } \\
\text { de transplantation }\end{array}\end{array}$ & $H 69$ & $51 \mathrm{~cm}$ & $27 \%$ & 21 & $39 \%$ \\
$\begin{array}{l}\text { Hauteur à 18 ans } \\
\begin{array}{l}(14 \text { ans de plantation) } \\
\text { Circonférence à 18 ans } \\
(14 \text { ans de plantation) }\end{array}\end{array}$ & $H 83$ & 88 & $20 \%$ & 57 & $24 \%$ \\
\hline
\end{tabular}

seulement du côté des fortes densités). Les 2 zones parallèles aux grands côtés du rectangle central contiennent chacune un des génotypes en plantation pure, mais avec le même gradient de densité que dans le dispositif proprement dit.

La variation de la densité totale $(N /$ ha) est proportionnelle aux abscisses: $N=166,66 \cdot X$ ( $X$ en mètres, origine extérieure).

Le pourcentage $P_{\mathrm{A}}$ des plants du génotype A est proportionnel aux ordonnées: $P_{A}=3,33$. $Y$ ( $Y$ en mètres). $N$ et $P_{A}$ sont évidemment des grandeurs théoriques.

La plantation a été faite en mai 1970. Les plants étaient âgés de 4 ans $(2+2)$. Les entretiens ont été réalisés par fauchage $(1970,1971)$ et traitement chimique (diquat + paraquat, 1971, 1972, 1973).

Les principales grandeurs mesurées sont la circonférence à $1,30 \mathrm{~m}$ et la hauteur totale. La variable prise en considération ici, notée $\mathrm{C83}$, est la circonférence à la fin de la saison de végétation de 1983. La hauteur au même âge n'a pu être utilisée pour cette étude car elle n'a pas été mesurée dans les peuplements purs et seulement pour 1 arbre sur 9 dans le peuplement mélangé.

Les 2 descendances ont des croissances nettement différentes comme le montrent les moyennes calculées pour chacune d'elles, toutes densitées et tous mélanges confondus (tableau I). On a étudié successivement les 2 types d'interaction cités en introduction. L'analyse statistique consiste en une comparaison des régressions $C 83$ sur. $N$, calculées pour chacun des 2 génotypes en plantation pure (interaction statistique) ou pour un même génotype mais à différents taux de mélange (interaction biologique). On teste le parallélisme des courbes de régression puis, si parallélisme il y a, leur coïncidence en comparant leur ordonnée à l'origine (Kozak 1970, Décourt 1971).
Indépendamment des facteurs expérimentaux, il existe une importante variabilité individuelle, liée à la répartition spatiale irrégulière et aussi à la variabilité initiale de la taille des plants ( $H 69$, hauteur à la plantation) ou à leur hauteur en fin de crise de transplantation ( $H 72$, fin de la période où la croissance en hauteur est médiocre). La variabilité spatiale n'a pu être prise en compte, faute d'une mesure de densité locale performante (Dreyfus, 1988). Les hauteurs à la plantation et en fin de crise de transplantation sont liées positivement à C83 (tableau II)

Pour H69, cette liaison statistique est partiellement biaisée à cause d'une tendance probablement inconsciente à utiliser, lors de la plantation, des plants de taille moyenne un peu supérieure pour les densités les plus faibles; or, celles-ci sont justement les plus favorables pour la croissance en circonférence. En outre, la crise de transplantation a occasionné de nombreux déclassements individuels; parmi les déclassements importants entre le rang de 1969 et le rang de 1983 (correspondant, par exemple, à un changement de rang d'une amplitude supérieure à $10 \%$ de l'effectif), plus des 2 tiers se sont produits entre 1969 et 1972 (données non publiées).

Pour ces 2 raisons, et bien que $H 72$ soit déjà influencée négativement par la densité $\left(R^{2}\right.$

Tableau II. Corrélations entre $\mathrm{C} 83$ et $\mathrm{H} 69$ ou H72

\begin{tabular}{ccc}
\hline & $\begin{array}{c}\text { GANN 7 } \\
\text { (725 arbres) }\end{array}$ & $\begin{array}{c}\text { MORZ 4 } \\
\text { (701 arbres) }\end{array}$ \\
\hline H69 & $\mathrm{R}^{2}=0,20$ & $\mathrm{R}^{2}=0,22$ \\
$H 72$ & $\mathrm{R}^{2}=0,37$ & $\mathrm{R}^{2}=0,24$ \\
\hline
\end{tabular}


$=0,060$ pour 725 individus GANN $7 ; \mathrm{R}^{2}=0,003$ pour 701 individus MORZ 4 ) et qu'il en résulte une légère sous-estimation de l'effet du gradient de densité sur $\mathrm{C83}$, on a préféré utiliser H72 plutôt que $\mathrm{H69}$ comme covariable de C83. La hauteur fin 1972 correspond à un stade où les effets incontrôlables de la crise de transplantation ont pratiquement cessé de jouer; moins d'un tiers des déclassements cités précédemment ont eu lieu entre 1972 et 1983.

L'utilisation d'une covariable permet de réduire la dispersion autour de l'ajustement de C83 à N.C83 a été corrigé à partir de la régression linéaire de C83 sur H72 calculée pour l'ensemble des individus de chaque génotype:

- GANN 7

\section{- MORZ 4}

$C 83_{i}=0,334 \cdot H 72_{i}-2,747+\varepsilon$

C83 $3_{\mathrm{i}}$ corrigé $=0,334 \cdot H 72 M-2,747+\varepsilon_{\mathrm{i}}$;

$C 83_{i}=0,278 \cdot H 72_{i}+2,268+\varepsilon_{i}$

$C 83_{i}$ corrigé $=0,278 \cdot H 72 M+2,268+\varepsilon_{i}$

pour $C 83_{\mathrm{i}}$ et $H 72_{\mathrm{i}}$ en $\mathrm{cm}$

$\varepsilon_{\mathrm{i}}=$ résidu

$H 72 M=$ moyenne arithmétique de $H 72$ pour tous les individus du génotype considéré.

La circonférence C83 corrigée correspond à une circonférence ramenée à hauteur $\mathrm{H} 72$ égale. Pour la suite, C83 corrigée est notée C83.

\section{Peuplements purs - Interaction statis- tique entre génotype et densité totale}

Du fait du caractère clinal du gradient de mélange, le génotype GANN 7 pur et le génotype MORZ 4 pur ne sont représentés dans le dispositif proprement dit que de manière théorique et linéaire sur les grands côtés du rectangle central. Dans le dispositif proprement dit, il n'existe pas de véritable peuplement pur. Pour comparer l'effet de la densité sur chacun des génotypes en peuplement pur, nous nous sommes résolus à utiliser les zones de protection. Comme celles-ci sont influencées par l'effet de lisière, les valeurs indiquées (200 à 10 000/ha) surestiment les densités réellement éprouvées, au moins pour les arbres de bordure; ceux-ci ont été conservés dans l'échantillon faute d'un critère satisfaisant pour les définir et les éliminer. L'étude concerne 286 individus GANN 7 et 292 individus MORZ 4.

On note sans surprise une taille moyenne (C83) nettement plus élevée pour GANN 7 pur que pour MORZ 4 pur et, pour les 2 génotypes, une réduction très forte de $C 83$ lorsque la densité augmente. Pour savoir si cette réduction est semblable pour les 2 descendances ou si, au contraire, il existe une interaction statistique entre le génotype et la densité du peuplement, on a comparé les 2 courbes $C 83=f(N)$.

Notons tout d'abord que pour les 2 peuplements purs, la relation entre $C 83$ et $N$ est mieux représentée par un modèle quadratique en $N$ et $N^{R}$ que par un modèle linéaire ou cubique. Les équations ci-dessous, correspondant aux 3 modèles comparés par l'analyse, permettent d'illustrer le fonctionnement de cette dernière: - premier modèle: courbe unique

(1) $C 83=42,6-5,02 \cdot 10^{-3} \cdot N+2,7 \cdot 10^{-7} \cdot N^{2}$ $\mathrm{SCE}=20340 ; \mathrm{ddl}=575 ; \mathrm{C} 83 \mathrm{en} \mathrm{cm}$;

- deuxième modèle: 2 courbes non coïncidentes mais parallèles

GANN $7: C 83=44,6-5,07 \cdot 10^{-3} \cdot N$

(2) $+2,8 \cdot 10^{-7} \cdot N^{2}$

MORZ 4: $C 83=40,3-5,07 \cdot 10^{-3} \cdot N$ $+2,8 \cdot 10^{-7} \cdot N^{2}$

SCE $=17643 ; \mathrm{ddl}=574$

- troisième modèle: 2 courbes non coïncidentes et non parallèles

(3)

GANN 7: $C 83=45,5-5,42 \cdot 10^{-3} \cdot N$ $+3,1 \cdot 10^{-7} \cdot N^{2}$

$$
\begin{aligned}
& \text { MORZ 4: C83 }=39,4-4,70 \cdot 10^{-3} \cdot N \\
& +2,5 \cdot 10^{-7} \cdot N^{2} \\
& \text { SCE }=17627 ; \mathrm{ddl}=572 .
\end{aligned}
$$

Les test $F$ indiquent que l'on peut considérer les 2 courbes comme étant parallèles mais pas coïncidentes:

- parallélisme: $F=[(17643-17$ 627) $/(574-$ $572)] /(17643 / 574)$

$=0,27 P=0,23$

- coïncidence: $F=\left[\left(\begin{array}{lll}20 & 340-17 & 643\end{array}\right) /(575-\right.$ $574)] /(20340 / 575)$

$=87,7 P=0,99$

Autrement dit, les 2 génotypes ne peuvent pas être réduits à l'équation unique (1), mais les 2 courbes correspondant aux équations du système (3) (fig. 2) n'ont pas des directions significativement différentes. Le système d'équations (2), intermédiaire, est statistiquement la meilleure représentation de l'influence de la densité sur la taille des arbres des 2 


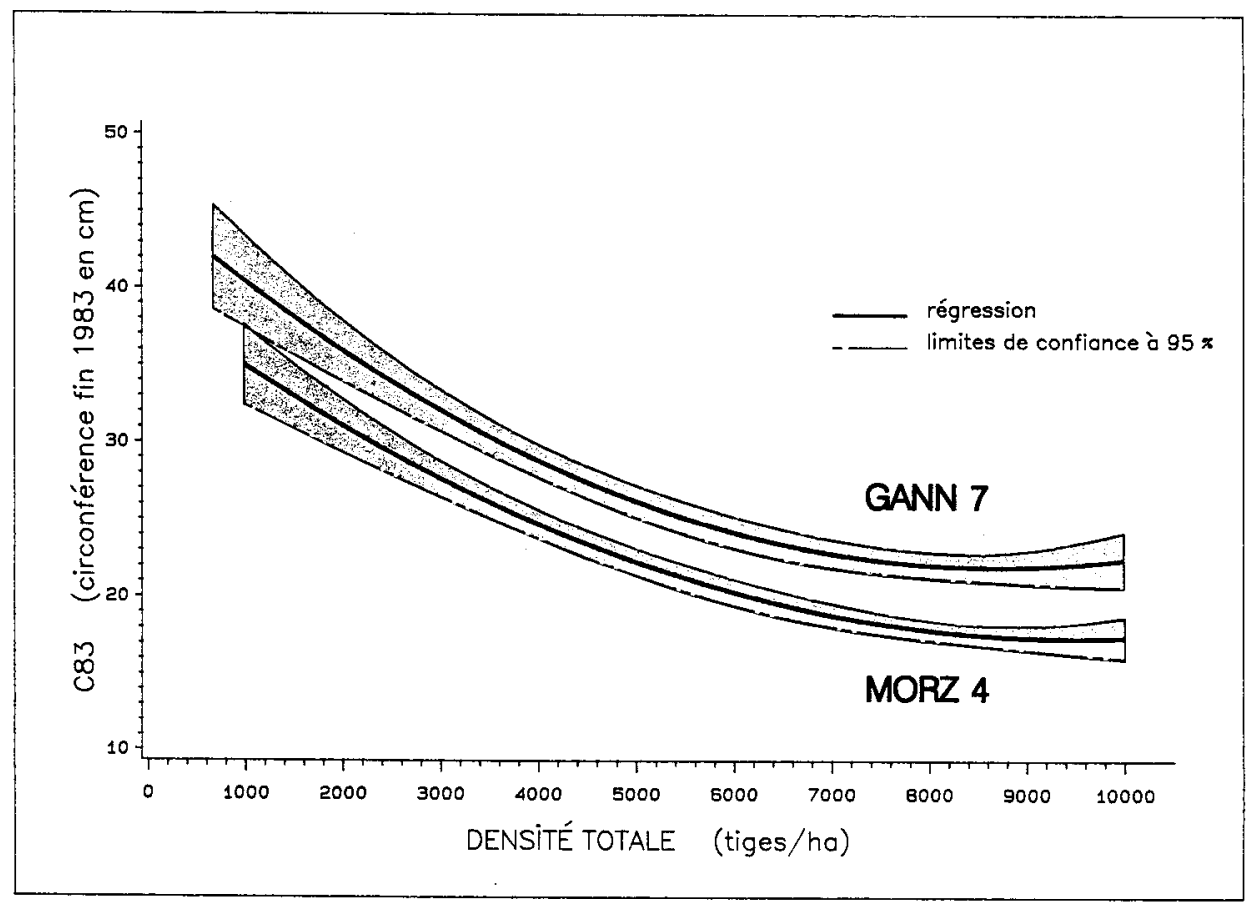

Fig. 2. Influence de la densité sur la circonférence en 1983 (C83) pour chaque génotype en peuplement pur. Intervalles de confiance pour les valeurs prédites moyennes au seuil de probabilité $P=0,95$.

génotypes pour notre échantillon: les courbes correspondant à GANN 7 pur et à MORZ 4 pur sont considérées comme étant parallèles. Pour ce système d'équations, l'écart type moyen résiduel est ramené à $5,5 \mathrm{~cm}$.

Le parallélisme des courbes semble indiquer que le facteur densité intervient de manière similaire sur la croissance en circonférence des deux génotypes en peuplement pur: sur la période considérée (14 ans depuis la plantation), une même augmentation de densité a provoqué une perte de croissance équivalente en valeur absolue pour les 2 génotypes (en valeur relative, la perte est donc plus forte pour MORZ 4).

Toutefois, compte tenu du «bruit de fond» important introduit par la position aléatoire des arbres dans le dispositif, on ne peut pas considérer qu'il n'existe pas d'interaction statistique; on peut simplement affirmer que si elle existe, elle est d'importance trop limitée pour être décelable dans cette expérimentation.

\section{Peuplements mélangés - Interaction biologique - Compétition intergénoty- pique}

\section{Approche descriptive}

Cette fois, l'étude porte sur le dispositif complet. La situation observée est décrite à la figure 3 . Bien que la densité et le mélange varient de façon continue, les tendances observées ne peuvent être traduites directement sous la forme d'une surface de réponse à cause de la grande variabilité des conditions de croissance pour une densité théorique donnée. Les tailles 


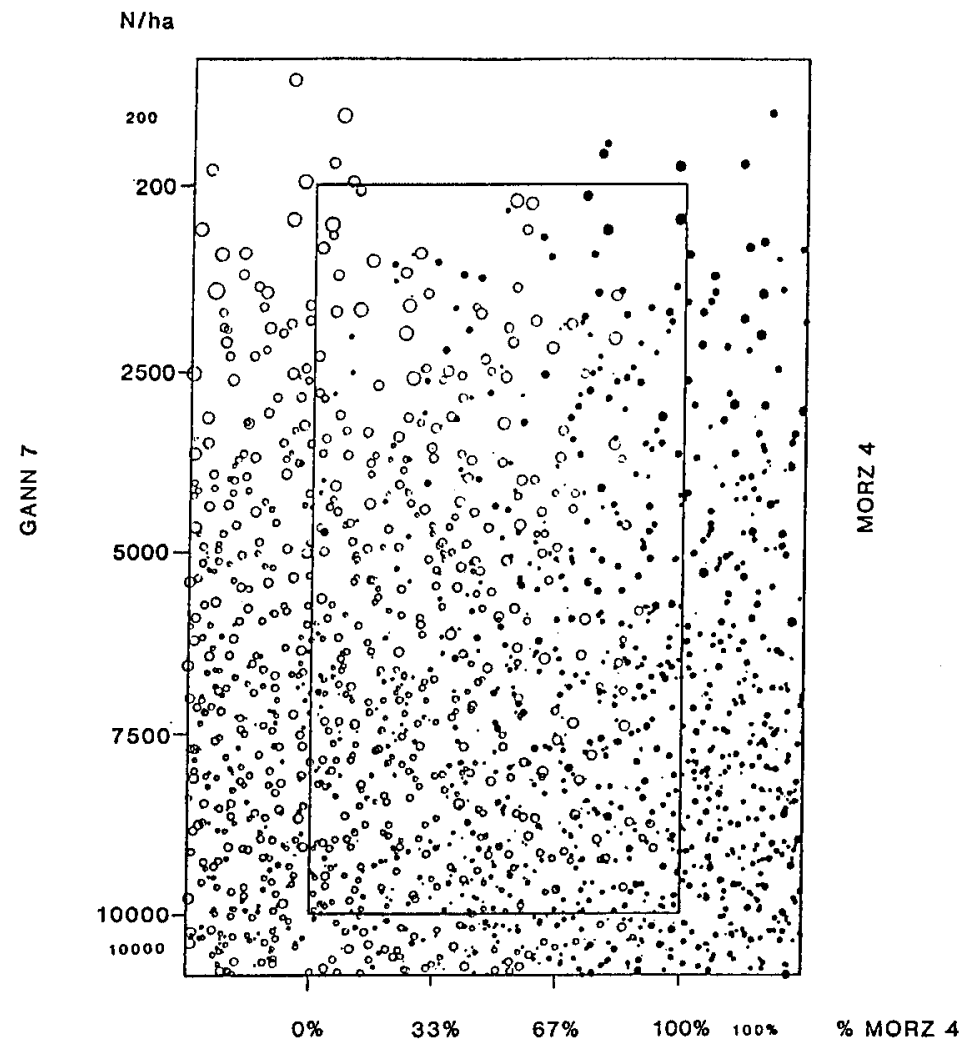

Fig. 3. Cartographie du dispositif et représentation des circonférences individuelles de 1983. Cercles vides: GANN 7, cercles pleins: MORZ 4, diamètre proportionnel à C83.

moyennes (C83) sont représentées de façon simplifiée à la figure 4 pour 5 tranches de densité définies arbitrairement, en peuplement pur et dans 3 situations de mélange:

- «67-100\%», mélange contenant au moins $2 / 3$ de plants du génotype considéré (le peuplement pur est exclu);

- «33-67\%», mélange contenant entre $1 / 3$ et 2/3 de plants du génotype considéré;

- «0-33\%», mélange contenant moins d' $1 / 3$ de plants du génotype considéré (le peuplement pur de l'autre génotype est évidemment exclu).

Schématiquement, on constate, d'une part qu'à densité totale égale, les arbres du génotype vigoureux (GANN 7) ont moins bien poussé en peuplement pur qu'en mélange avec les arbres du génotype peu vigoureux (MORZ 4), et d'autre part, que ces derniers ont mieux poussé en peuplement pur que confrontés au génotype vigoureux. Ceci correspond à une interaction de compétition entre les 2 génotypes.

Pour une densité donnée, la croissance d'un arbre, quel que soit son génotype, a été d'autant meilleure qu'il se trouvait dans une partie du dispositif où la proportion de MORZ 4

Tableau III. Effectifs par génotype pour chaque situation de mélange.

\begin{tabular}{lcc}
\hline Effectif & Gann 7 & Morz 4 \\
\hline Pur & 286 & 292 \\
$67-100 \%$ & 251 & 241 \\
$33-67 \%$ & 152 & 135 \\
$0-33 \%$ & 36 & 33 \\
\hline
\end{tabular}



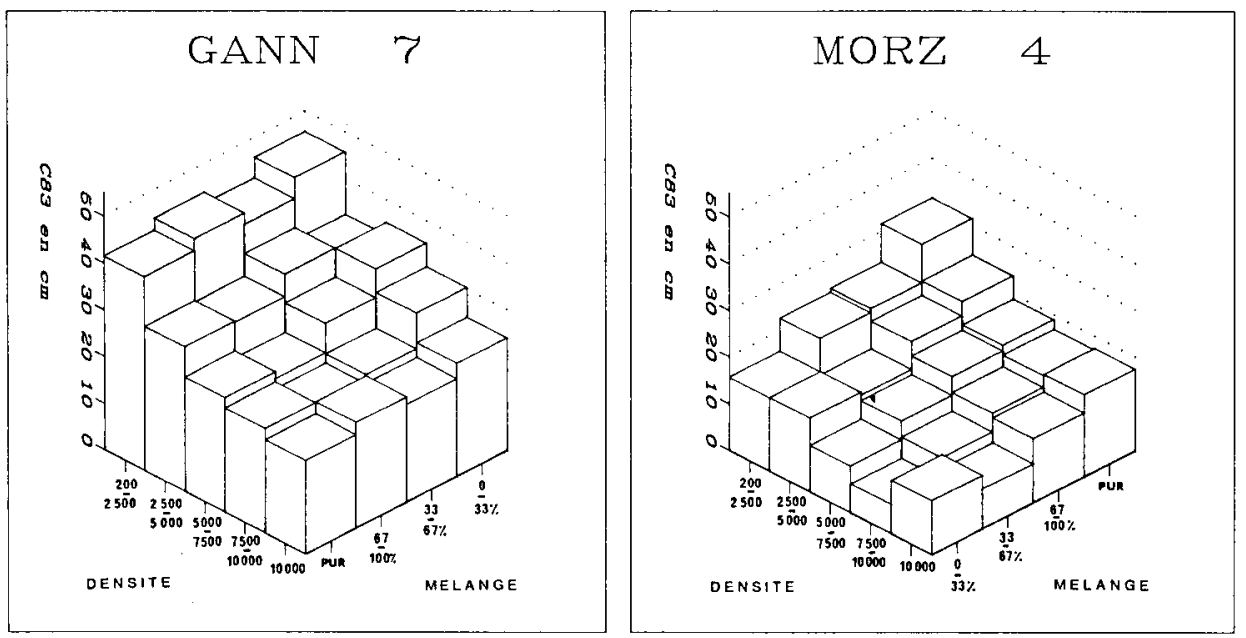

Fig. 4. Circonférence en 1983 (C83) de chaque génotype pour 5 tranches de densité et 4 proportions de mélange (y compris le peuplement pur).

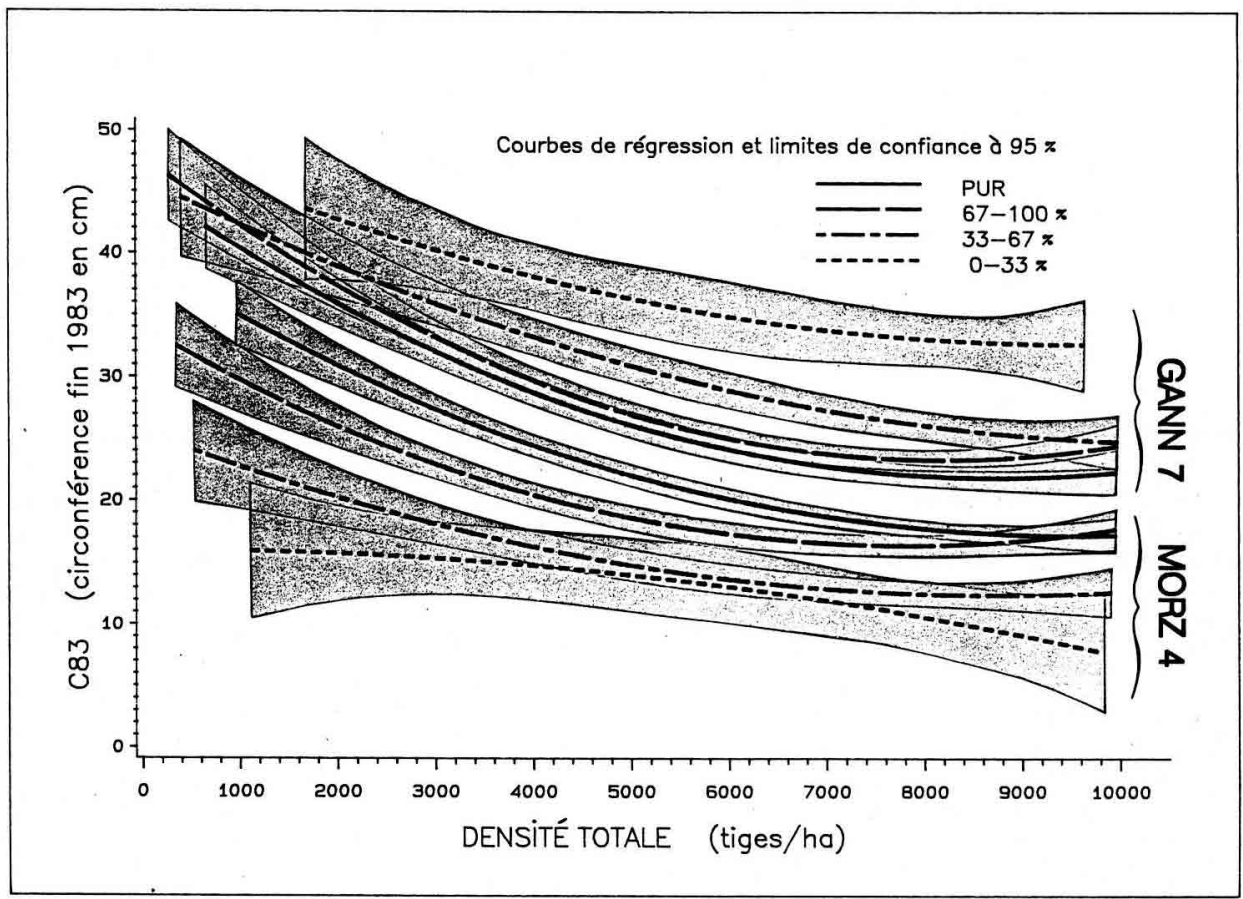

Fig. 5. Courbes de régression de la circonférence en 1983 (C83) sur la densité totale (GANN $7+$ MORZ 4), en peuplement pur et pour 3 proportions de mélange. Intervalles de confiance pour les valeurs prédites moyennes au seuil de probabilité $P=0,95$. 
Tableau IV. GANN 7: Tests du parallélisme et de la coïncidene des courbes de régression $C 83=f$ (densité totale).

\begin{tabular}{|c|c|c|c|}
\hline GANN 7 & Pur & $67-100 \%$ & $33-67 \%$ \\
\hline $67-100 \%$ & $\begin{array}{c}537 \\
F p=0,28 P p=0,24 \\
F c=6,12 P C=0,99\end{array}$ & & \\
\hline $33-67 \%$ & $\begin{array}{c}438 \\
F p=0,83 \mathrm{Pp}=0,56 \\
F \mathrm{C}=41,6 \mathrm{PC}=0,99\end{array}$ & $\begin{array}{c}403 \\
F p=1,93 \mathrm{Pp}=0,85 \\
F c=19,1 \quad \mathrm{Pc}=0,99\end{array}$ & \\
\hline $0-33 \%$ & $\begin{array}{c}322 \\
F p=0,69 P p=0,50 \\
F C=90,3 \quad P C=0,99\end{array}$ & $\begin{array}{c}287 \\
\mathrm{Fp}=0,80 \mathrm{Pp}=0,55 \\
\mathrm{Fc}=75,7 \mathrm{Pc}=0,99\end{array}$ & $\begin{array}{c}188 \\
\mathrm{Fp}=0,59 \mathrm{Pp}=0,44 \\
\mathrm{Fc}=31,3 \mathrm{PC}=0,99\end{array}$ \\
\hline
\end{tabular}

Tableau V. MORZ 4: Tests du parallélisme et de la coïncidence des courbes de régression $C 83=f$ (densité totale).

\begin{tabular}{lccc}
\hline MORZ 4 & Pur & $67-100 \%$ & $33-67 \%$ \\
\hline $67-100 \%$ & 533 & & \\
& $F p=6,47 \mathrm{Pp}=0,99$ & & \\
& $\mathrm{FC}=22,2 \mathrm{Pc}=0,99$ & & \\
$33-67 \%$ & 427 & $\mathrm{Fp}=0,89 \mathrm{Pp}=0,59$ & \\
& $\mathrm{Fp}=5,55 \mathrm{Pp}=0,99$ & $\mathrm{Fc}=58,5 \mathrm{Pc}=0,99$ & 168 \\
& $\mathrm{Fc}=152 \mathrm{Pc}=0,99$ & 274 & $\mathrm{Fp}=1,65 \mathrm{Pp}=0,81$ \\
$0-33 \%$ & & & \\
& $\mathrm{Fp}=6,24 \mathrm{Pp}=0,99$ & $\mathrm{Fp}=3,71 \mathrm{Pp}=0,97$ & $\mathrm{Fc}=5,86 \mathrm{PC}=0,98$ \\
\hline
\end{tabular}

était plus forte (et donc la proportion de GANN 7 plus réduite). MORZ 4 possède par conséquent un pouvọir concurrentiel plus faible que celui de GANN 7, ce qui concorde avec les caractéristiques de vigueur des 2 génotypes.

\section{Analyse statistique}

On compare pour un génotype donné les courbes de régression de $C 83$ sur $N$ correspondant au peuplement pur et aux 3 situations de mélange citées.

En premier lieu, on a cherché à savoir si la relation entre C83 et $N$ était linéaire en $N$ ou curvilinéaire en $N, N$. Lorsque le génotype est pur ou représente plus des $2 / 3$ du mélange, la meilleure régression est celle en $N$ et $N^{2}$. Lorsqu'il représente moins d'un tiers ou entre un tiers et deux tiers du mélange, le modèle en $N$ et $N^{2}$ n'est pas meilleur que le modèle linéaire. Mais la taille des échantillons dont on dispose est considérablement plus faible pour les 2 derniers cas que pour le génotype pur ou représentant plus des $2 / 3$ du mélange (tableau III). II est fort probable qu'un effectif plus important aurait permis de conclure à une allure curvilinéaire. Partant de cette hypothèse, et par souci d'homogénéité, nous avons adopté le modèle curvilinéaire en $N$ et $N^{2}$ pour les 4 situations (peuplement pur et 3 taux de mélange).

Ces 4 situations sont comparées 2 à 2 , et on teste le parallélisme et la coïncidence des courbes correspondantes afin de savoir si la confrontation avec l'autre génotype influence seulement la taille moyenne ou si elle influence également la façon dont la densité totale agit sur la circonférence 1983. Les courbes corres- 
pondant aux 4 situations sont représentées à la figure 5 pour MORZ 4 et pour GANN 7 avec les intervalles de confiance pour les valeurs prédites moyennes au seuil de probabilité $P=$ 0,95 ; certaines courbes débutent à une densité un peu supérieure à 200/ha parce que l'échantillon correspondant ne contient pas d'arbres plus proches de 200/ha. Les résultats des tests statistiques sont donnés tableaux IV et $\mathrm{V}$. Dans chaque case, qui correspond à la comparaison de 2 courbes, figurent l'effectif concerné, le test $\mathrm{F}$ et le seuil de probabilité pour le parallélisme $(F p, P p)$ et éventuellement pour la coïncidence ( $\mathrm{Fc}, \mathrm{Pc}$ ).

Dans le cas de MORZ 4, même si les courbes correspondant aux mélanges n'ont pas toutes des allures significativement différentes (cas de «0-33\%» et «33-67\%», de «33-67\%» et de «67-100\%»), aucune n'est parallèle à la courbe correspondant au peuplement pur. Pour ce génotype, il existe donc une interaction statistique entre la compétition avec GANN 7 et la densité du mélange.

Pour GANN 7 , en revanche, l'analyse ne détecte pas de différence significative en ce qui concerne la forme des courbes. II semble néanmoins que les diverses situations se distinguent à la fois par des différences de taille moyenne et par la rapidité de la décroissance de C83 en fonction de la densité: la concurrence se ferait sentir plus rapidement en peuplement pur qu'en mélange à prépondérance de MORZ 4. La variabilité des conditions de croissance liée au caractère aléatoire du dispositif masque probablement en partie les différences d'allure entre les courbes, différences qui, de ce fait, ne sont pas détectées par l'analyse. De plus, les trois mélanges sont définis par des fourchettes et non par un taux précis d'où une variabilité des conditions au sein même d'un échantillon, variabilité qui nuit à la distinction des mélanges.

Il est intéressant d'examiner plus en détail le comportement des deux génotypes:

- a) aux faibles densités, les courbes sont proches les unes des autres pour GANN 7 et nettement éloignées pour MORZ 4. A ces densités, GANN 7 profite donc peu de la confrontation avec un génotype moins vigoureux. Aux très grands espacements, la compétition est faible de toute façon; elle influence peu la croissance de GANN 7. Par contre, pour MORZ 4, le mélange correspond au remplacement d'arbres peu vigoureux par des compétiteurs beaucoup plus "agressifs», et la compétition intergénotypique se fait sentir dès les faibles densités;

- b) lorsque la densité augmente, l'influence de la compétition intergénotypique s'accentue pour GANN 7 et s'atténue un peu pour MORZ 4. Pour GANN 7, le remplacement d'individus du même génotype par des concurrents moins vigoureux est d'autant pius profitable que la pression globale de concurrence s'accentue. Pour MORZ 4, à forte densité, la taille est très réduite quel que soit le taux de mélange. La confrontation avec GANN 7 reste très défavorable mais, en valeur absolue les différences sont moins importantes qu'à faible densité;

- c) la remontée de certaines courbes pour des densiliés voisines de 10 000/ha correspond probablement à un effet de lisière. La zone de protection à l'extrémité la plus dense du dispositif n'a que $5 \mathrm{~m}$ de largeur pour une hauteur moyenne en 1983 de $4,0 \mathrm{~m}$ pour MORZ 4 et de $7,4 \mathrm{~m}$ pour GANN 7 dans la tranche de densité 7500 10000 ; elle ne suffit apparemment pas à absorber l'effet favorable en bordure du peuplement;

Tableau VI. GANN7: Test du parallélisme et de la coïncidence des courbes de régression $C 83=f$ (densité de GANN 7).

\begin{tabular}{|c|c|c|c|}
\hline Gann 7 & Pur & $67-100 \%$ & $33-67 \%$ \\
\hline $67-100 \%$ & $\begin{array}{c}537 \\
F p=0,73 \mathrm{Pp}=0,52 \\
F \mathrm{C}=0,01 \mathrm{PC}=0,07\end{array}$ & & \\
\hline $33-67 \%$ & $\begin{array}{c}438 \\
F p=0,54 P p=0,42 \\
F c=9,11 P c=0,99\end{array}$ & $\begin{array}{c}403 \\
F p=0,52 \mathrm{Pp}=0,40 \\
F C=6,05 \mathrm{PC}=0,99\end{array}$ & \\
\hline $0-33 \%$ & $\begin{array}{c}322 \\
\mathrm{Fp}=0,72 \mathrm{Pp}=0,51 \\
\mathrm{FC}=4,39 \mathrm{PC}=0,96\end{array}$ & $\begin{array}{c}287 \\
F p=0,85 P p=0,57 \\
F C=4,11 P C=0,96\end{array}$ & $\begin{array}{c}188 \\
F p=1,46 \mathrm{Pp}=0,77 \\
\mathrm{FC}=3,51 \mathrm{PC}=0,94\end{array}$ \\
\hline
\end{tabular}


Tableau VII. MORZ 4: Tests du parallélisme et de la coïncidence des courbes de régression C83 = $f$ (densité de MORZ 4).

\begin{tabular}{|c|c|c|c|}
\hline MORZ 4 & Pur & $67-100 \%$ & $33-67 \%$ \\
\hline $67-100 \%$ & $\begin{array}{c}533 \\
F p=15,1 P p=0,99 \\
F C=40,2 P C=0,99\end{array}$ & & \\
\hline $33-67 \%$ & $\begin{array}{c}427 \\
F p=10,6 P p=0,99 \\
F c=293 P C=0,99\end{array}$ & $\begin{array}{c}376 \\
F p=4,92 \mathrm{Pp}=0,99 \\
F \mathrm{C}=94,2 \mathrm{PC}=0,99\end{array}$ & \\
\hline $0-33 \%$ & $\begin{array}{c}325 \\
F p=2,24 P p=0,89 \\
F c=197 P c=0,99\end{array}$ & $\begin{array}{c}274 \\
F p=1,90 P p=0,85 \\
F C=98,3 P c=0,99\end{array}$ & $\begin{array}{c}168 \\
F p=1,19 P p=0,69 \\
F C=14,4 P C=0,99\end{array}$ \\
\hline
\end{tabular}

- d) pour GANN 7, les courbes «peuplement pur» et « $67 \%-100 \%$ s sont très voisines, au moins jusqu'à une densité totale de $6000 / h a$. Ceci peut résulter de l'emploi de la zone de protection en tant que peuplement pur: l'effet de lisière provoque probablement une remontée de la courbe "peuplement pur», ce qui tend à la rapprocher de la courbe $« 67 \%-100 \% »$. Peutêtre ceci signifie-t-il aussi que la tension de compétition se relâche peu lorsque le rempla-

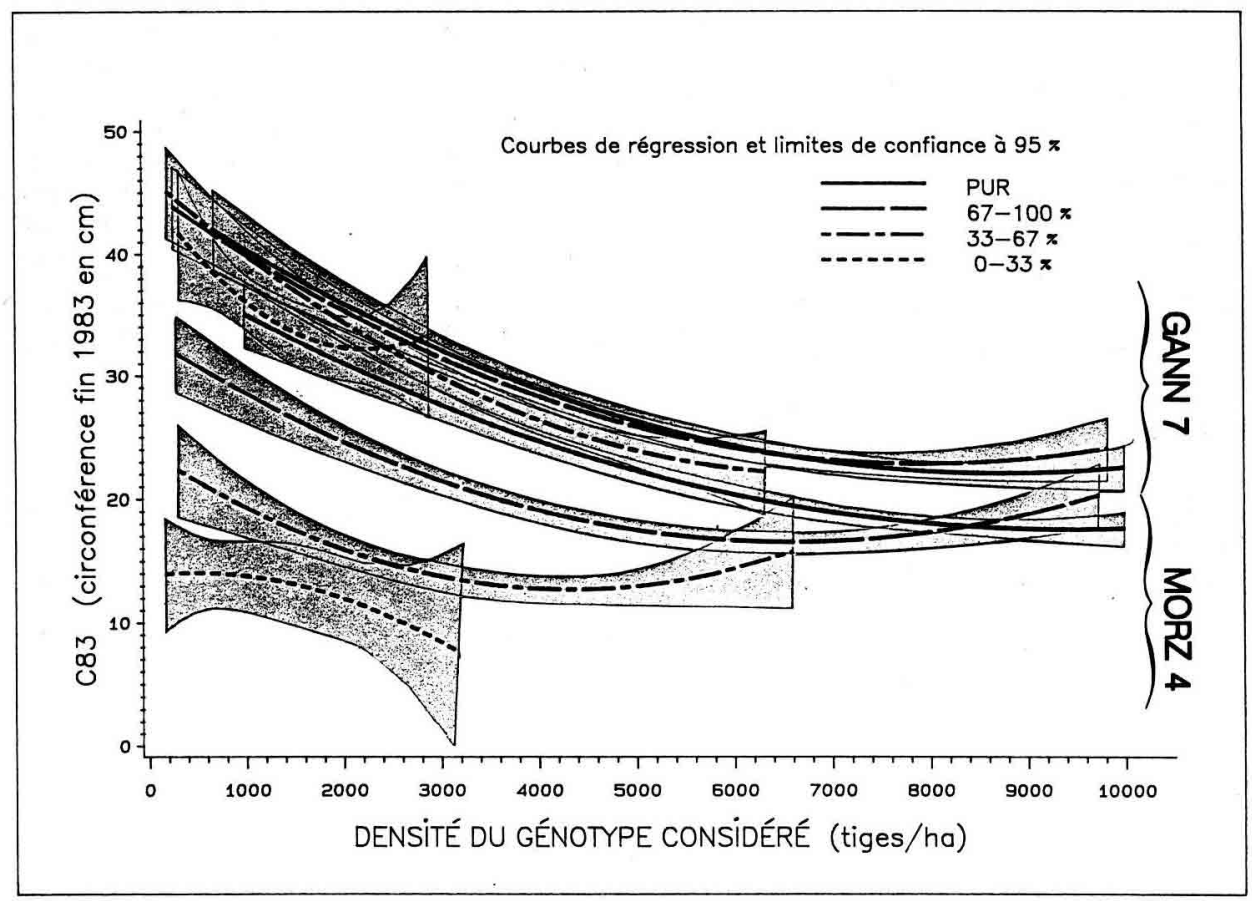

Fig. 6. Courbes de régression de la circonférence en 1983 (C83) sur la densité du génotype considéré (GANN 7 ou MORZ 4), en peuplement pur et pour 3 proportions de mélange. Intervalles de confiance pour les valeurs prédites moyennes au seuil de probabilité $P=0,95$. 
cement par des individus moins vigoureux ne porte que sur une faible partie de l'effectif total, mais ce n'est qu'une hypothèse;

- e) en ce qui concerne MORZ 4, on constate que la concavité de la courbe «0\%-33\%» est inversée. En fait, comme on l'a signalé plus haut, l'effectif de l'échantillon correspondant est très faible et cette courbe correspondant à la tranche $33-67 \%$. L'analyse ne détecte d'ailleurs pas de différence significative d'allure entre ces 2 courbes.

\section{Nature et importance de l'interaction biologique}

On peut conclure à une influence nette des proportions du mélange sur la croissance des 2 descendances maternelles pour une densité donnée (interaction biologique, ou compétition intergénotypique) et à une influence probable du mélange sur la réaction au facteur densité (interaction statistique entre densité et compéti- tion intergénotypique), même si, pour GANN 7, cette dernière interaction n'est pas statistiquement significative dans cette expérience.

Pour préciser la nature de ces interactions, on a refait les analyses en prenant comme variable explicatrice, non plus la densité totale, mais la densité du seul génotype concerné (fig. 6). La densité d'un génotype varie respectivement de $\approx 50$ à 2355 , de 100 à 5000 et de $\approx$ 150 à 7355 selon qu'il représente moins de $1 / 3,1 / 3$ à $2 / 3$ ou $2 / 3$ à $100 \%$ du mélange, et évidemment de 200 à 10000 pour le peuplement pur. Les intervalles de confiance de la moyenne au seuil de probabilité $P=0,95$ sont représentés; les résultats des tests statistiques sont donnés dans les tableaux VI et VII.

L'examen des 4 courbes correspondant à la descendance GANN 7 montre que MORZ 4 exerce malgré tout à son égard une action concurrentielle: à l'inverse du faisceau des courbes tracées en fonction de la densité totale (fig. 5), les courbes les plus hautes correspondent ici aux peuplements où GANN 7 est le

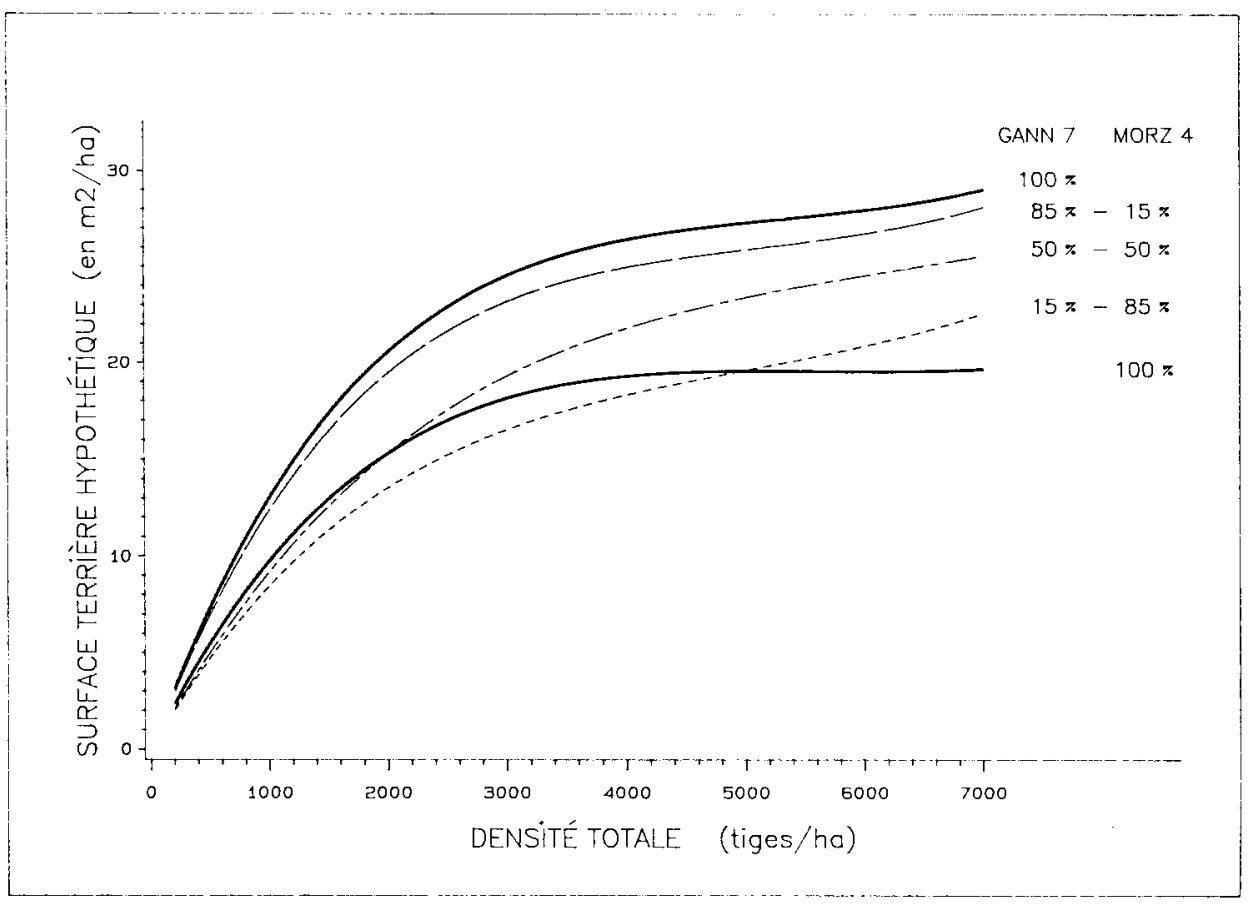

Fig. 7. Simulation de la surface terrière en fonction de la densité pour des peuplements purs de GANN 7 ou MORZ 4 et pour 3 peuplements mélangés. 
mieux représenté. Bien que les tests de parallélisme et de coïncidence des courbes ne revèlent pas de différence significative, il est indiscutable que GANN 7 ne profite pas réellement de la présence de MORZ 4. Pour un nombre fixe d'individus du génotype GANN 7, l'ajout d'individus MORZ 4 (en l'occurrence, un complément à concurrence de la densité totale) paraît pénaliser la croissance individuelle moyenne de GANN 7.

II s'agit par conséquent d'une interaction compétitive classique, c'est-à-dire d'une concurrence. La plus forte croissance des individus GANN 7 situés dans une partie à dominance de MORZ 4 n'est due qu'à la faiblesse de la concurrence exercée par MORZ 4 et non pas à un phénomène de coopération. En ce qui concerne MORZ 4, le faisceau de courbes ne fait que confirmer la sensibilité de cette provenance à la concurrence exercée par GANN 7: les 4 courbes sont considérablement plus écartées que pour ce dernier.

On a tenté d'apprécier l'impact de la compétition intergénotypique sur la production en surface terrière au niveau du peuplement. Les régressions $C 83=f(N)$ réalisées précédemment permettent de calculer, en fonction de la densité, une estimation simple de la surface terrière de 5 peuplements fictifs correspondant aux 2 plantations pures (de GANN 7 et de MORZ 4) et à 3 plantations mélangées où GANN 7 représenterait 15,50 et $85 \%$ de l'effectif et MORZ 4 le complément à 100\%.

Les courbes sont présentées pour des densités de 200/ha à 7 000/ha (fig. 7); au-delà, les estimations deviennent trop incertaines car les effets de lisière constatés à l'extrémité des courbes $C 83=f(M)$ sont amplifiés par le cumul des valeurs individuelles. II apparaît qu'aucun mélange n'est supérieur au peuplement pur de GANN 7. Du fait de la structure particulière du dispositif dont les équations sont tirées, il ne faut pas voir dans ce résultat plus qu'une tendance conforme à celles signalées dans la littérature et qui mériterait d'être confirmée dans des peuplements en grandeur réelle, de densité et de composition homogènes.

\section{DISCUSSION}

L'étude de ce dispositif très original suscite quelques réflexions sur le plan méthodologique. Dans tout dispositif destiné à l'étude de ces phénomènes, il serait intéressant d'avoir une plus forte représentation des densités auxquelles sont cultivés les peuplements dans la pratique $(<1100-3300 / \mathrm{ha})$. Sans réduire forcément la fourchette des densités expérimentées ici, et en partant du schéma expérimental élaboré par Décourt (1970), ceci pourrait être obtenu en adoptant un effectif constant pour chaque densité, grâce à une variation non linéaire de la densité le long de la parcelle. En outre, il paraît indispensable de disposer de peuplements purs permettant une comparaison tout à fait rigoureuse avec les mélanges.

La distribution aléatoire des arbres est à l'origine d'une forte variabilité des conditions de croissance. Si elle présente par ailleurs certains avantages (Décourt, 1970), cette variabilité rend difficile la détermination de la relation entre la densité et la croissance, alors que cette détermination est assez facile dans un dispositif clinal régulier, par exemple de type Nelder (Illy, Lemoine, 1970; Décourt, Lemoine, 1974; Panetsos, 1980; Lemoine, 1980). En théorie, le recours à une caractérisation de la densité locale permet de pallier cet inconvénient mais on ne dispose pas, à l'heure actuelle, de mesures de densité locale suffisamment efficaces, ni de connaissances biologiques (en particulier concernant la compétition racinaire) permettant d'en élaborer une (Dreyfus, 1988). Un dispositif du même type mais non aléatoire pour la densité serait sans doute préférable pour cette analyse: par exemple, une plantation régulière dont l'espacement varierait de façon clinale, le mélange pouvant rester distribué de façon clinale et aléatoire.

En expérimentation, on a généralement intérêt à maîtriser le mieux possible les sources de variabilité. L'utilisation de clones dans les dispositifs expérimentaux permettrait de réduire fortement la variabilité intragénotypique. Elle contribuerait en outre à combler le manque de connaissances sylvicoles pour les clones considérés. 
La sylviculture intensive moderne s'oriente en effet de plus en plus vers l'utilisation d'un matériel très sélectionné, y compris les clones. Pour limiter les risques liés à la fragilité sanitaire des peuplements monoclonaux, il est envisagé, soit de créer des peuplements multiclonaux (les clones d'épicéa commun commercialisés actuellement ne le sont que sous forme de mélanges de plusieurs centaines de clones), soit de juxtaposer des petites parcelles monoclonales et d'organiser une rotation des clones à chaque renouvellement.

Bien qu'il n'ait pas bénéficié d'un matériel aussi finement sélectionné, non disponible pour l'épicéa à l'époque de sa mise en place, le dispositif expérimental que nous avons étudié a permis de montrer que peuplements pur et mélangés peuvent correspondre, pour un génotype donné, à des lois de croissance différentes et probablement à des tests de sélection différents. L'existence ou l'absence d'interaction statistique génotype-densité est importante dans la perspective d'une sylviculture de peuplements monoclonaux. La compétition intergénotypique et son interaction avec la densité doivent, quant à elles, être prises en compte dans la sélection de génotypes destinés à des plantations multiclonales.

L'absence d'interaction statistique décelable entre le génotype et la densité pour la croissance en circonférence va dans le même sens que les résultats obtenus par Birot (1972) pour des semis de Douglas en pépinière. Si cette situation se confirmait pour d'autres génotypes, y compris des clones, dans des dispositifs mieux adaptés que celui étudié ici, cela permettrait d'obtenir rapidement des courbes de croissance applicables pour les génotypes nouvellement sélectionnés. Dans une telle optique, il serait nécessaire de rechercher aussi des interactions éventuelles pour d'autres caractères, en particulier la croissance en hauteur.
Le maintien de la supériorité d'un des génotypes sur l'autre, quelles que soient les conditions de mélange et de densité, confirme les conclusions de Campbell et Wilson (1973). Même si elle correspond à un déséquilibre connu a priori, la forte compétition intergénotypique observée ici illustre les difficultés inhérentes à la culture de peuplements mélangés. Pour les descendances maternelles étudiées, la croissance des peuplements mélangés ne paraît pas pouvoir atteindre, ni a fortiori dépasser, celle du peuplement pur du génotype le plus vigoureux.

\section{CONCLUSION}

Les phénomènes mis en évidence sont en concordance avec ceux couramment observés en matière forestière:

- en peuplement pur, la densité influence de façon assez semblable la croissance de génotypes apparentés, même s'ils sont de vigueurs différentes;

- l'aptitude à la compétition intergénotypique paraît être liée très étroitement à la vigueur.

II faut cependant remarquer que les génotypes étudiés sont encore relativement variables et que les rares observations en contradiction avec les constats indiqués ci-dessus ont été réalisées sur des clones de peupliers (Delvaux, 1967; Panetsos, 1980). Des clones sont maintenant disponibles pour des essences forestières de plus en plus nombreuses et on peut imaginer qu'avec ce type de matériel végétal, on pourra mettre en évidence expérimentalement des interactions dont on pourrait alors tirer parti sur le plan pratique.

\section{REMERCIEMENTS}

Je remercie F Ningre qui m'a fourni les informations nécessaires pour l'étude de ce dispositif, 
D Betored et $M$ Turrel qui ont participé à la réalisation des figures, Mlle $\vee$ Farhat pour la dactylographie, $H$ Oswald qui a accepté de me faire part de ses remarques lors de la rédaction de cet article.

\section{RÉFÉRENCES}

Birot Y (1972) Densité de semis, effets de compétition et variabilité intraspécifique chez le Douglas en pépinière. Ann Sci Forest 29, 403-426

Birot $Y$ (1986) Principaux apports de la génétique et de l'amélioration des arbres forestiers à la sylviculture. Situation actuelle et perspectives. Rev For $\mathrm{Fr}^{\circ}{ }^{\circ}$ spécial, 38, 208220

Campbell RK, Wilson BC (1973) Spacing-genotype interaction in Douglas fir. Silvae Genet $22,15-20$

Décourt N (1970) Utilisation des dispositifs clinaux pour l'étude de la compétition dans les peuplements forestiers. Ve Colloque d'écologie, 12-14 mars 1970, ENS, Paris. INRA, CR Nancy, Station de sylviculture et de production. Document interne $n^{\circ} 70-71$

Décourt N (1971) Comparaison des équations de régression. Application au cubage des peuplements d'épicéa commun. Ann Sci Forest 28, 51-58

Décourt N, Lemoine M (1974) Premiers résultats d'une expérience clinale d'espacement avec Populus trichocarpa. Ann Sci Forest $31,171-179$

Delvaux J (1967) Contribution à l'étude de l'éducation des peuplements. V. Compétition inter-clones chez le peuplier. VI. Action d'écrans multilatéraux translucides et opaques sur le peuplier. VII. Compétition inter-individuelie chez le peuplier. VIII. Effets de lisière dans les plantations de peuplier. Station de recherches des eaux et forêts Groenendaal-Hoeilaart, Belgique. Travaux, Série $B, 33$

De Wit CT (1960) On competition. Versl Landbouwk Onderz (Agric Res Rep) 66, 8

Dreyfus $P$ (1988) Compétition et croissance dans de jeunes peuplements résineux. Aspects biologiques, écologiques et sylvicoles. Thèse de doctorat en sciences, Université d'Aix-Marseille-III

Illy G, Lemoine B (1970) Densité de peuplement, concurrence et coopération chez le pin maritime. I. - Premiers résultats d'une plantation à espacement variable. Ann Sci Forest 27, 127-155

Kozak A (1970) A simple method to test parallelism and coincidence for curvilinear, multiple linear and multiple curvilinear regressions. Compte-rendu. IUFRO - $3^{e}$ Conférence du Groupe consultatif des statisticiens forestiers, Jouy-en-Josas, 7-11 septembre. INRA, pp. $133-145$

Lemoine B (1980) Densité de peuplement, concurrence et coopération chez le pin maritime. II. - Résultats à 5 et 10 ans d'une plantation à espacement variable. $\mathrm{Ann} \mathrm{Sci}$ Forest 37, 217-237

Panetsos CP (1980) Selection of new poplar clones under various spacings. Silvae Genet, 29, 130-135

Perry DA (1986) The competition process in forest stands. Department of Forest Science, Oregon State University, Corvallis, USA. Communication faite à Edimgbourg 